\title{
Estudio del análisis e interpretación de un problema matemático evaluados a estudiantes universitarios de primer año (2019) con la perspectiva didáctica en la existencia de la solución
}

Study of the analysis and interpretation of the solution of a mathematical problem evaluated to first-year university students (2019) with the didactic perspective on the existence of the solution

Recibido: noviembre 20 de 2019 | Revisado: diciembre 12 de 2019 | Aceptado: enero 25 de 2020

Neisser Pino Romero ${ }^{\mathrm{I}}$

Sonia Alanya PÉrez ${ }^{\mathrm{I}}$

1 Universidad Peruana Cayetano Heredia neisser.pino@upch.pe

\begin{abstract}
RESUMEN
El presente estudio trata sobre el desarrollo de un problema matemático (ecuaciones aplicadas a edades) dentro del área del algebra a nivel secundaria. Donde se añadió la concepción de algunas formalidades matemáticas como corresponde al nivel universitario que está dentro de las exigencias a los estudiantes de primer año de formación (Estudios Generales). El problema aplicado a los estudiantes se enfoca en el análisis de la posible respuesta de acuerdo al enunciado de la misma, y generar un debate sobre el modelamiento matemático de un problema real expresado en ecuaciones matemáticas.
\end{abstract}

Palabras clave: educación Matemática, existencia de soluciones, modelamiento matemático, formulación y resolución de ecuaciones, interpretación de soluciones

\begin{abstract}
In the present work, a study will be made on the development of a mathematical problem (equations applied to ages) within the area of algebra at the secondary level. Where the conception of some mathematical formalities will be added as corresponds to the university level that is within the requirements of the first year students of formation (General Studies). Where the problem applied to the students focuses on the analysis of the possible answer according to the statement of the same, and generate a debate about the mathematical modeling of a real problem expressed in mathematical equations.
\end{abstract}

Key words: Mathematics education, existence of solutions, mathematical modeling, formulation and resolution of equations, interpretation of solutions

(C) Los autores. Este artículo es publicado por la Revista Campus de la Facultad de Ingeniería y Arquitectura de la Universidad de San Martín de Porres. Este artículo se distribuye en los términos de la Licencia Creative Commons Atribución No-comercial - Compartir-Igual 4.0 Internacional (https://creativecommons.org/licenses/ CC-BY), que permite el uso no comercial, distribución y reproducción en cualquier medio siempre que la obra original sea debidamente citada. Para uso comercial contactar a: revistacampus@usmp.pe. 


\section{Introducción}

El análisis de los problemas matemáticos aplicados a la vida real es un tema muy abordado durante el colegio, especialmente, en la etapa de la secundaria donde después de terminar, se deberían tener los conocimientos básicos y mínimos para ingresar a la universidad (Aparicio \& Bazán, 1997). La matemática, como un área de formación escolar, permite al alumno abstraerse y comprender un enunciado para formularlo en alguna ecuación, y desde aquí, obtener una solución y dar respuesta a la pregunta del mismo enunciado (Flores, 2014). Según (Rodriguez, 2016) este procedimiento es vital para su crecimiento cognitivo $y$ en su desarrollo integral que forma parte de su vida diaria. Por lo cual, cuando se empiezan los cursos de matemática en la universidad tienen un enfoque analítico de acuerdo a la formación académica que exige la misma carrera profesional. En este sentido, la exigencia de problemas analíticos más que operativos es la perspectiva de un nuevo lineamiento de la formación universitaria de acuerdo a la nueva Ley Universitaria (Ministerio de Educación, 2014) donde la metodología es inseparable de los supuestos teóricos, el problema y los propósitos de la investigación; alude a la manera en que se trata el problema de investigación y a los procedimientos para buscar las respuestas (Taylor \& Bodgan, 1992). Por consiguiente, los enfoques de los problemas matemáticos deben ser de una visión más allá de una simple operación. (Malaspina Jurado, 2016)

\section{Formulación del problema matemático}

En esta sección, se presenta el enfoque del problema matemático que se utilizó como herramienta para evaluar el impacto que genera en el razonamiento de alumnos universitarios de primer año de formación. Se escogió un problema de ecuaciones relacionado a edades debido que es un tema bastante conocido en la escuela y es una base para la interpretación de un problema real que se puede expresar matemáticamente, obtener una respuesta, dar una interpretación lógica y que va con el contraste de la realidad del problema enunciado. (Aparicio, 2006)

Cabe decir que la formulación de problemas en los cursos universitarios de matemática tiene un enfoque más analítico y con una formalización del procedimiento del problema. Se puede expresar este procedimiento en el siguiente esquema propuesto por (Tan Soo, 2018) en su libro Matemáticas Aplicadas a los Negocios, las Ciencias Sociales y la Vida, y también lo expresa (Pino, 2017) en su artículo enfocado a la Matemática como herramienta para entender la Economía y la Economía como fuente de problemas matemáticos dando a comprender la importancia de un correcto y estricto modelaje de los problemas de la vida real, y consolidando una formación científica y humanística en el estudiante universitario con una formación para el desarrollo profesional adecuado que la sociedad exige (Vilchez, 2007).

En la Figura 1, se puede describir cada etapa en la resolución de un problema real mediante un modelamiento matemático según los siguientes autores (Baldor, 1941), 
(Malaspina Jurado, 2016), (Pino, 2017) y (Tan Soo, 2018) como una estructura adecuada de comprensión para una abstracción de un problema real y poder plantearlo en un modelo matemático. Es decir: (i) Formular, dado un problema real, la primera labor es formular el problema con un lenguaje matemático.

Las numerosas técnicas utilizadas en la construcción de modelos matemáticos van desde la consideración teórica del problema hasta la interpretación de los datos asociados con el problema. Por consiguiente, la mayoría de los modelos matemáticos implican funciones de una o más variables $\mathrm{o}$ ecuaciones que las definan (implícitamente). (ii) Resolver, una vez construido el modelo matemático, se puede utilizar técnicas matemáticas apropiadas, las cuales permitirán solucionarel problema. (iii) Interpretar, después de haber obtenido la solución matemática que se adecúa al modelo matemático, se tiene que interpretar los resultados en el contexto del problema real original. (iv) Probar, algunos modelos matemáticos de aplicaciones reales describen situaciones con una precisión completa. Pero otros modelos matemáticos dan, en lo mejor de los casos, una descripción aproximada del problema real. En este caso tener que probar la precisión del modelo observando qué tan bien describe el problema real y predice el comportamiento pasado o futuro. Si estos resultados no son satisfactorios, entonces puede que se tenga que volver a considerar los supuestos hechos en la construcción del modelo o, en el peor de los casos, iniciar de nuevo la construcción del modelo como se realiza en el paso Formular.

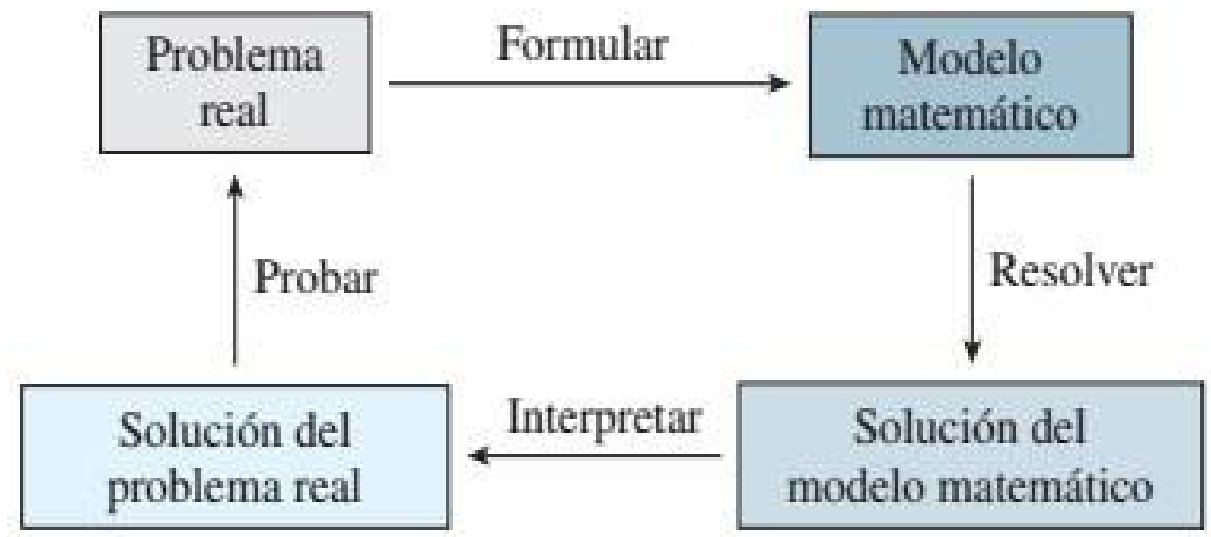

Figura 1. Construcción de un modelo matemático

Nota. Matemáticas Aplicadas a los Negocios, las Ciencias Sociales y la Vida, Tan (2018)

Realmente, se presenta un proceso ordenado, sistematizado y coherente para un adecuado algoritmo de resolución de un problema real, y cómo la matemática ayuda a resolver los problemas de la vida real es la herramienta idónea para la resolución de problemas. (Malaspina Jurado, 2016).
Ahora presentamos dos ejemplos de problemas matemáticos con respecto a las edades. Y se muestra cómo usualmente se resuelven en la etapa escolar, y hasta en la etapa universitaria. (Chuquilin Cubas, 2011) 
Problema 1: Si dentro de 10 años Adriana tiene el triple de la edad que tiene ahora,

¿qué edad tendrá entonces?

Solución: Llamamos $\boldsymbol{x}$ a la edad actual de Adriana. Como Adriana tiene ahora $\boldsymbol{x}$ ańos, dentro de 10 ańos su edad será $\boldsymbol{x}+$ 10. El triple de la edad que tiene ahora es $\mathbf{3} \boldsymbol{x}$. Por tanto, la ecuación que expresa que dentro de 10 ańos la edad será el triple que la actual es: $x+10=3 x$, donde $\boldsymbol{x}=\mathbf{5}$. Luego, la edad actual de Adriana es 5 años. Dentro de 10 ańos, su edad será 15 años.

Problema 2: La abuela de Lucía tiene cinco veces su edad y su madre tiene la mitad de edad que su abuela. Dentro de seis años, la edad de la Lucía es la mitad que la de su madre, ¿qué edad tiene cada una?

Solución: Si la edad de Lucía es $\boldsymbol{x}$, la de su abuela es $\mathbf{5} \boldsymbol{x}$, y la de su madre $\frac{\mathbf{2}}{2} \boldsymbol{x}$

La ecuación que tenemos es:

$$
x+6=\frac{1}{2}\left(\frac{5}{2} x+6\right) \text {, donde } \boldsymbol{x}=\mathbf{1 2} \text {. }
$$

Luego, Lucía tiene 12 años, su madre tiene $\mathbf{3 0}$ años y su abuela tiene $\mathbf{6 0}$ años.

Como se ha podido observar en estos dos problemas sencillos, los estudiantes usualmente formulan la ecuación correctamente, y desarrollan la ecuación hasta encontrar la solución. Y luego, se expresa la respuesta al problema de acuerdo a las preguntas formuladas. Pero, surge una pregunta fundamental que no se realiza de manera explícita en el problema, ni se hace mención al menos en la formación secundaria pero en la superior debería considerarse de forma fundamental para un adecuado análisis del problema. El alumno se pregunta en algún momento, ¿el problema siempre tiene solución? (Rodriguez, 2016).

\section{Formalización del problema (edades)}

Ahora formularemos algunos detalles que generalmente nadie considera en la resolución de un problema. Como mencionaba el escritor Conan Doyle en su libro: No hay nada más engañoso que un hecho evidente. Ante este hecho, la matemática ha ido formalizando cada paso de la resolución debido que todo proceso matemático está gobernado por axiomas y propiedades inquebrantables que garantizan una estructura bien definida. (Zapata \& Blanco, 2009).

La matemática presenta conceptos de formalización en la resolución de un problema de la vida real donde la consideración de todo el problema bien definido se centra en lo siguiente: la Existencia de Soluciones y la Unicidad de Soluciones, en algunos problemas donde lo exija la misma formulación de las variables, se considerará la Positividad de las Soluciones. Estas consideraciones parecerían no muy importantes en la resolución de los problemas debido que se presumen apriori que el problema lo satisface, $y$ ante este hecho, se procede a la resolución del problema de forma casi inmediata. (Malaspina Jurado, 2016).

En los problemas de edades, los estudiantes deberían al menos considerar estos detalles, no porque ayuden a la resolución del problema en su aspecto operativo y funcional, sino más bien en la aprehensión y comprensión del problema, y cómo la matemática ayuda a resolver problemas de mayor 
complejidad. Y de manera específica, en la formalización de los problemas de edades, se debería considerar el espacio de las soluciones, es decir, dentro de qué espacio de los números se encontrará la solución, debido que el enunciado del problema lo indica de manera puntual o de forma implícita.

Un problema de edades contiene variables que se asigna a cada personaje que se incluya en el enunciado. Desde aquí, estas variables deben ser no negativas, es decir, positivas. Por lo cual, el espacio de solución inicial a considerar sería: $\mathrm{R}_{0}{ }^{+}$, donde también se considera el valor cero porque representa el inicio de la vida. Pero si uno analiza con mayor detalle, la medición de la edad en la vida cotidiana, se indica con números enteros $(Z)$, pero con el mismo detalle de la no negatividad se debe especificar el espacio correspondiente sería los números naturales $(\mathrm{N})$ donde también se incluya al número cero. Este proceso de razonamiento permite una mejor concepción de la solución antes de construir la ecuación y resolver la ecuación. Lo cual, es el proceso adecuado para un estudiante universitario que busca un análisis más completo para afrontar la problemática de la vida real. (Pino, 2017).

Esta parte teórica de la matemática aplicada a los problemas de la vida real se centra en algunas consideraciones triviales que pasan sin mucha importancia al momento de resolver problemas, pero contienen un fundamento básico para la comprensión y abstracción del problema y cómo resolverlo debidamente de tal forma que brinda soluciones mejor fundamentadas, siendo todo este razonamiento ordenando y lógico, la piedra angular de la formación universitaria, para una consolidación del aprendizaje integral.

\section{Enunciado del problema para los estudiantes}

Presentamos el problema que se evaluó a los estudiantes de primer semestre académico. Se desarrolló detalladamente el problema propuesto para poder enfocar el estudio en los estudiantes. Este problema se puede considerar un ejercicio muy abundante en el análisis matemático y en la comprensión pedagógica que se puede desprender después de la evaluación hacia los estudiantes. (Flores, 2014).

El problema que se utilizó ha sido recopilado de una película espańola titulada "La Habitación de Fermat" (2007), y se ubica en el acertijo 8 del transcurso de la película. Se escogió este debido que el enunciado puede generar confusión y controversia ante el planteamiento de un problema matemático cuando se va definiendo las variables que conformarán la ecuación. (Ecured, 2008)

\section{Problema Propuesto (Una Cuestión de Edades)}

Una madre es 21 años mayor que su hijo. Al cabo de seis ańos la edad de la madre será cinco veces la que tenga el hijo. ¿Qué está haciendo el padre?

Del problema propuesto, se puede construir las siguientes consideraciones iniciales.

- Unidad temporal: años

- Espacio de soluciones: valores no negativos $\left(\mathrm{N}_{0}\right)$ 
Observación 1: Donde el valor del cero indica el nacimiento o el inicio de la vida de la persona, donde también se considera el punto referencial al tiempo Presente.

Observación 2: Surge la pregunta sobre la variable del Padre, debido que no hay datos relacionados a él. Causando la principal interrogante de este problema, más aún, ocasiona la crisis de encontrar solución a este problema. Por lo cual, se procede a la formulación de las ecuaciones para encontrar alguna solución de acuerdo al enunciado del problema.

Ahora, definimos las variables respectivas de la ecuación de acuerdo al orden que aparecen en el enunciado del problema, y además, la unidad temporal de todas las variables está en años.

- Madre : M (edad en años)

- Hijo : H (edad en años)

- Padre : P (edad en años)

Un adecuado reconocimiento que se puede inferir del enunciado es el estado del tiempo (pasado, presente y futuro), donde es importante definir adecuadamente la expresión matemática para la formulación de la ecuación, y así, poder obtener la solución. (Zapata \& Blanco , 2007).

- Tiempo Presente : $M=21+H$

- Tiempo Futuro : $M+6=5(H+$ 6)

Introduciendo la ecuación del tiempo presente a la ecuación del tiempo futuro obtenemos una sola ecuación con una sola variable. Teniendo en cuenta que todo está en la unidad temporal de años, tendremos que: $((21+H)+6)$ $=5(H+6)$, donde $\boldsymbol{H}=-\mathbf{3} / \mathbf{4}$. Después de obtener esta solución, se sitúa en una contradicción de lógica matemática debida que ya se había definido el espacio de soluciones (valores no negativos).

Una observación importante en la resolución de muchos problemas algebraicos aplicados, es la unidad de la dimensión de la variable, en nuestro caso, el tiempo (años). Bajo esta consideración la solución obtenida, se puede transformar a otra unidad temporal. Como se sabe, un año equivale 12 meses en la unidad de tiempo donde se encuentra el problema propuesto.

De aquí se puede transformar la solución obtenida con la intención de encontrar la respuesta final al problema propuesto, donde parece que es imposible encontrar de una manera directa $y$ tradicional de encontrar soluciones. $\mathrm{La}$ solución obtenida está expresada en años, pero también se puede representar bajo la unidad temporal mensual. Esto quiere decir, $\boldsymbol{H}=\mathbf{- 9}$ (meses). Aún después de la obtener la solución expresada en años, y luego de transformar la solución a meses, no se puede encontrar una respuesta para la pregunta del problema. Este punto en el análisis del resultado viene a ser el punto de quiebre y la apertura a un análisis aún mayor, debido que el estudiante debe relacionar la pregunta con la solución obtenida, y así dar una respuesta al problema. (Zapata \& Blanco, 2009).

La solución final (en meses) relacionado a la edad del Hijo, cuyo valor es de -9 meses, nos indica algo sobre el estado del tiempo en donde se encuentra, el cual es el Tiempo 
Presente, y una consideración que se tiene es el tiempo positivo. Aparte que no guarda una relación con la variable del Padre, y más aún, con la pregunta del problema, ¿Qué está haciendo? Pero desde esta comprensión del estado del tiempo, permite abstraer la relación del tiempo y la variable del Padre; la cual es que si $\boldsymbol{H}=\mathbf{0}$ significa el nacimiento del Hijo (bien en años o meses). Entonces el valor $\boldsymbol{H}=\mathbf{- 9}$ meses indicaría la concepción del Hijo (Tiempo Pasado), bajo con la consideración de un tiempo de gestación normal (nueve meses). Por ende, siguiendo este razonamiento, sí se puede responder lo qué está baciendo el Padre. Y la respuesta al problema sería: el Padre está en pleno coito con la Madre.

Después de terminar con la resolución detallada del problema donde se indica las directrices que tiene la resolución del mismo, y más aún, con una pregunta que en su primera impresión indicaría que no está relacionado con el enunciado de las hipótesis, y por ende, no se podría hallar respuesta. (Gutiérrez, 2011).

\section{Aplicación del problema a los estudiantes}

Esta sección se concentra en la descripción de la población de estudiantes evaluados con el problema propuesto en la subsección 2 para nuestro estudio experimental. Como se había mencionado, la población estuvo constituida por estudiantes de primer año de formación universitaria de la ciudad de Lima. (Cuena , Carrillo, De los Ríos, Reátegui, \& Ortiz, 2017). Se ha realizado la evaluación a un total de 450 estudiantes de tres universidades (una pública y dos privadas) de la ciudad de Lima. Todos los estudiantes cursaban el primer semestre académico de su formación universitaria. En el estudio no se ha hecho diferencia sobre las carreras que están estudiando como tampoco la edad ni el sexo, ni el tiempo de preparación adicional a la escolar (secundaria). La presentación y la evaluación del problema se suscitaron durante el desarrollo del curso de matemática como un problema especial de ecuaciones. De esta forma, se busca evaluar a los estudiantes de una forma espontánea y sin presiones para poder analizar sus expresiones, comentarios y observaciones ante el problema.

\section{Análisis del desarrollo del problema por los estudiantes}

Esta parte se centró en el estudio de las diferentes reacciones que generó por parte de los estudiantes con respecto al enunciado dictado oralmente del problema, el proceso de planteamiento del problema y obtención de una solución, y finalmente la obtención de la respuesta a la pregunta del problema propuesto. (Asensios, 2016).

\section{Resultados preliminares}

Se presentan dos gráficas que se relacionan entre sí, debido que la introducción de la variable Padre causó conmoción al momento de interpretar el problema propuesto debido a que se consideró leer (tres veces) el enunciado del problema hacia los estudiantes para resaltar el análisis de comprensión auditiva y su respectivo planteamiento de ecuaciones en los estudiantes. 

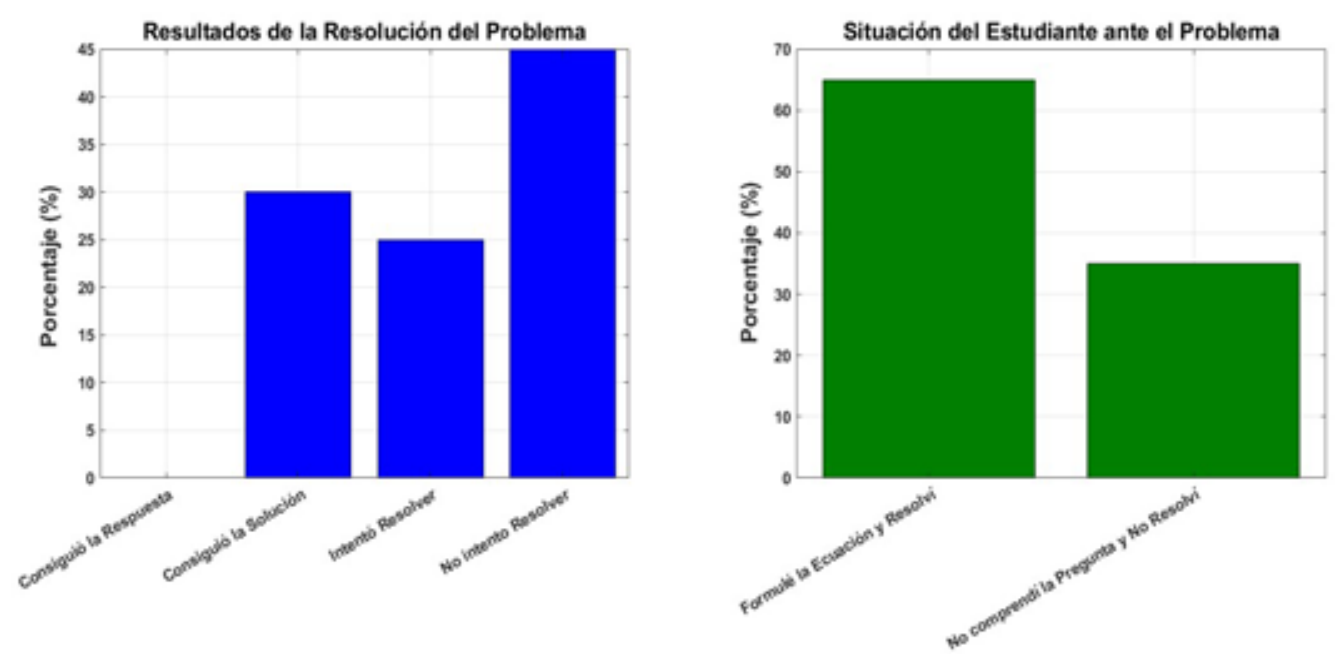

Figura 2. Impresión inicial ante el enunciado del problema

En la Figura 2, específicamente de la izquierda, se puede apreciar las reacciones de los estudiantes y sus respectivas acciones ante el problema propuesto donde un $45 \%$ de la población estudiantil no intentó resolverlo por quedarse pensando en la pregunta relacionada al padre mientras que un $25 \%$ intentó hacerlo, pero no obtuvo la solución de su ecuación o no pudo formular alguna ecuación, y finalmente el $30 \%$ sí consiguió la solución correcta $(\boldsymbol{H}=-\mathbf{3} / \mathbf{4})$, pero no dio la respuesta al problema.

En este sentido, los resultados se complementan con la gráfica de la derecha en cuanto a un hecho que ha marcado en los estudiantes, donde un 65\% formuló la ecuación y la resolvió (no importó la respuesta correcta, pero sí se halló la solución correcta) mientras que el 35\% indicó que no comprendió la pregunta y no la resolvió por ese motivo. Una percepción inicial ante un enunciado peculiar de un problema de edades, donde quizás sería la primera vez que tenían que desarrollar un problema con una pregunta que difiere a las hipótesis del enunciado (Jopen, Gómez, \& Olivera , 2014).
Después del tiempo establecido para la resolución del problema (15 minutos), se pasó a recoger las hojas de resolución, y luego de realizar un pequeńo cuestionario sobre el problema evaluado para los estudiantes. Esta consideración se realizó primero, antes de resolver, y luego para explicar el procedimiento que se debió realizar (como en la subsección 2. Este hecho, de explicar paso a paso, es la parte fundamental del estudio realizado en los estudiantes, debido a que cuestiona el análisis cotidiano de un problema de edades. Este desarrollo se realizó en la pizarra con la ayuda de los estudiantes. Para ello, se efectuó una formalización de las variables y las ecuaciones consideradas para la resolución. (Zapata \& Blanco, 2009) El hecho de explicar y fundamentar las bases elementales de la construcción del espacio de las soluciones y la definición de las variables.

Por tanto, la formalización de la ecuación donde se relaciona Madre e Hijo para obtener la solución, ha sido de vital importancia ya que que los estudiantes confirmaban la adecuada comprensión del problema planteado debido a que el porcentaje de estudiantes 
que no intentó resolver (45\%) estuvo atento a la explicación, mientras que el $55 \%$ se mantuvo expectanteexpectante de la respuesta ante la pregunta del problema. Con estos dos escenarios se puede analizar la diferencia de enfoque pedagógico para los dos grupos y así explicar, con detalle cómo se infiere la respuesta final del problema, y fomentar un mejor razonamiento y comprensión de las respuestas de los problemas aplicados. Cuando se dio la respuesta a la pregunta, fue el punto de quiebre de una respuesta que no se infería a partir de las hipótesis de forma directa, sino más bien, de una forma indirecta, $y$ ante este fundamental detalle, los estudiantes comprendieron que los problemas exigen una mayor comprensión. (INEI, 2018)

\section{Resultados finales}

Ante la resolución del problema que se realizó paso a paso, se fue identificando

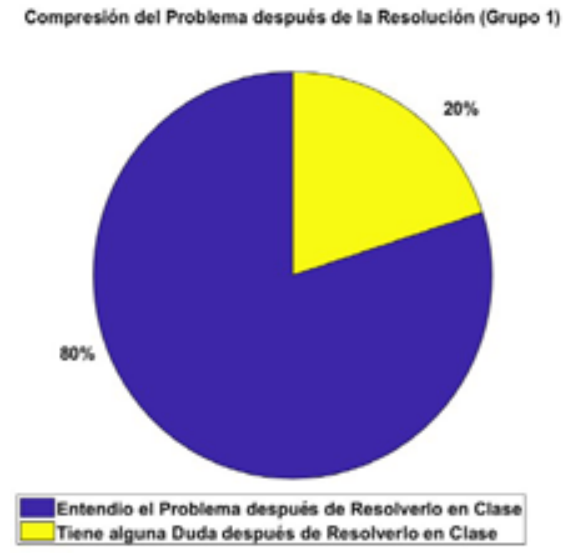

el dominio de la solución del problema, y luego la formulación, y finalmente la obtención de la solución. Y luego, se explicó la transformación de la unidad temporal para generar una línea de tiempo donde se pueda expresar la idea de modelización de la edad de una persona (tiempo positivo y usualmente de valor entero) con lo cual, se terminó la explicación del problema propuesto ante la sorpresa de los estudiantes. De aquí, se repartió nuevamente una pequeña hoja para qué respondieran unas preguntas, y así terminar el estudio en el aula de forma satisfactoria. (Zapata \& Blanco, 2007). En la última etapa de la aplicación, que los estudiantes escriban opiniones sobre el problema y la dinámica de la resolución del problema propuesto, para lo cual, esta intervención ellos se dividió en dos grupos (los que no habían obtenido la solución correcta $(H=-3 / 4)$ y los que la habían obtenido la solución) para su respectivo análisis de opiniones.

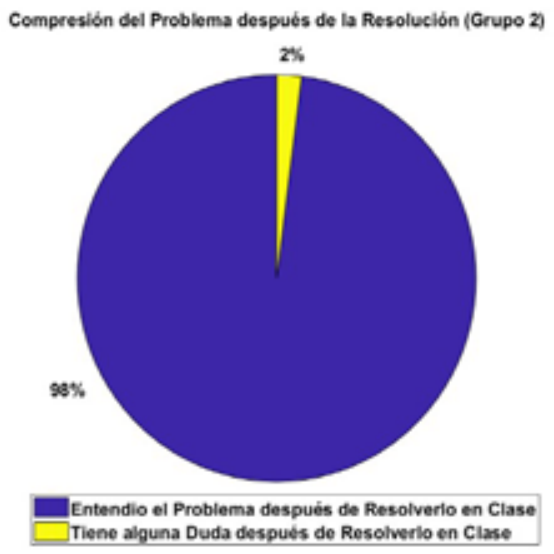

Figura 3. Nivel de comprensión de los estudiantes

Después de realizar el análisis sobre la compresión de la resolución y la forma adecuada para obtener la respuesta final ante la pregunta; se vio que un adecuado análisis de formalización y construcción de las variables y la ecuación ayuda mucho para la resolución del problema. Se preguntó a los estudiantes, y se obtuvo que el primer grupo que representaba el grupo que no habían obtenido la solución (gráfica de la izquierda) comprendió un $80 \%$ y solamente un $20 \%$ tiene alguna duda de la resolución mientras que el segundo grupo que eran los estudiantes que habían obtenido la solución (gráfica de la derecha) se obtuvo un $98 \%$ y 
solamente un 2\% tiene alguna duda de la resolución. Logrando una mejor comprensión ante una pregunta que inicialmente no parecería que no tuviera nada con el enunciado del problema (Figura 3).

Este estudio mediante muestra lo que se ha dicho en varias ocasiones sobre el nivel analítico de los estudiantes. De forma similar se expresó en el artículo "Actitudes hacia las Matemáticas en Ingresantes a la Universidad Nacional Agraria, La Molina” (Aparicio \& Bazán, 1997) y mediante un proceso estructurado como lo indica el profesor Malaspina en su artículo "Creación de problemas: sus potencialidades en la enseñanza y aprendizaje de las Matemáticas" (2016) nos han brindado una orientación adecuada para un

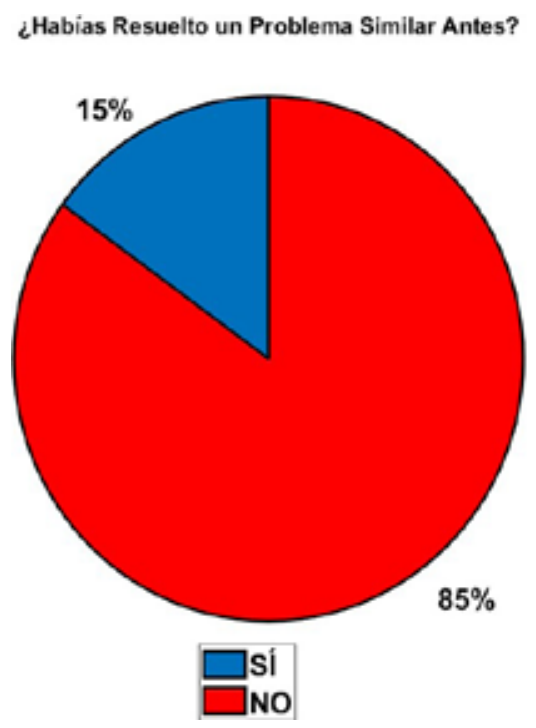

enfoque didáctico y analítico hacia los estudiantes de un simple problema algebraico (cuestión de edades). El estudio ha sido muy enriquecedor para los investigadores, pero sobre todo para los estudiantes ya que se brindó una perspectiva de análisis más allá de la técnica de desarrollo habitual.

En la última parte del estudio, se realizó nuevamente un formulario con preguntas sobre la aplicación de este problema en relación a su formación previa (colegio) y la formación que están recibiendo en la universidad actualmente, de tal forma que se puedan analizar sus conocimientos y experiencias previas para un problema que parecería diferente a los conocidos por ellos. Se realizaron diversas preguntas que analizamos a continuación. (Rodríguez, 2012).

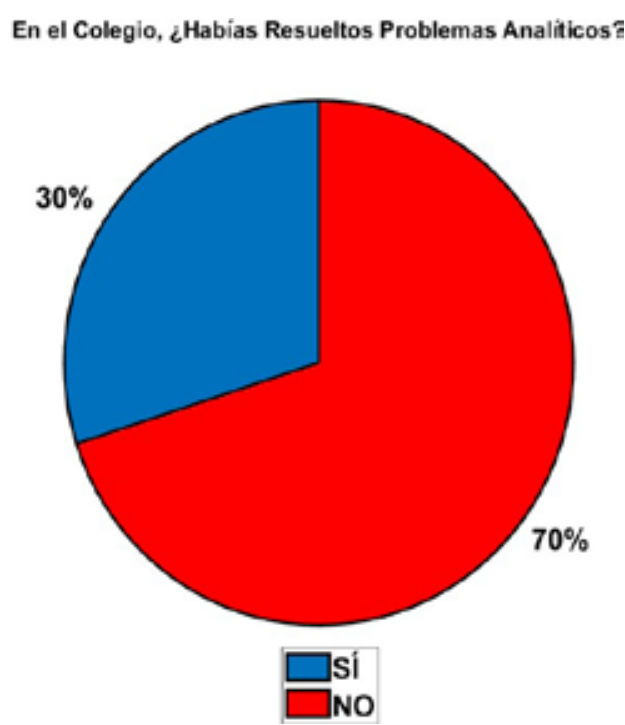

Figura 4. Antecedentes de los estudiantes ante el tipo de problema propuesto.

El análisis de las mismas nos permite deducir que la formación de la secundaria estaría enfocada a resolver problemas de un tipo más sencillo de análisis donde se pueda encontrar soluciones (problema matemático) y respuestas (problema real) a los problemas propuestos (Figura 4).
Este hecho ha resaltado mucho sobre el impacto que se puede suscitar en la formación universitaria. Por lo cual, en esta línea se realizaron dos preguntas más para visualizar cómo ellos pueden interpretar la realidad mediante el modelamiento matemático. (Arias, 2005) 

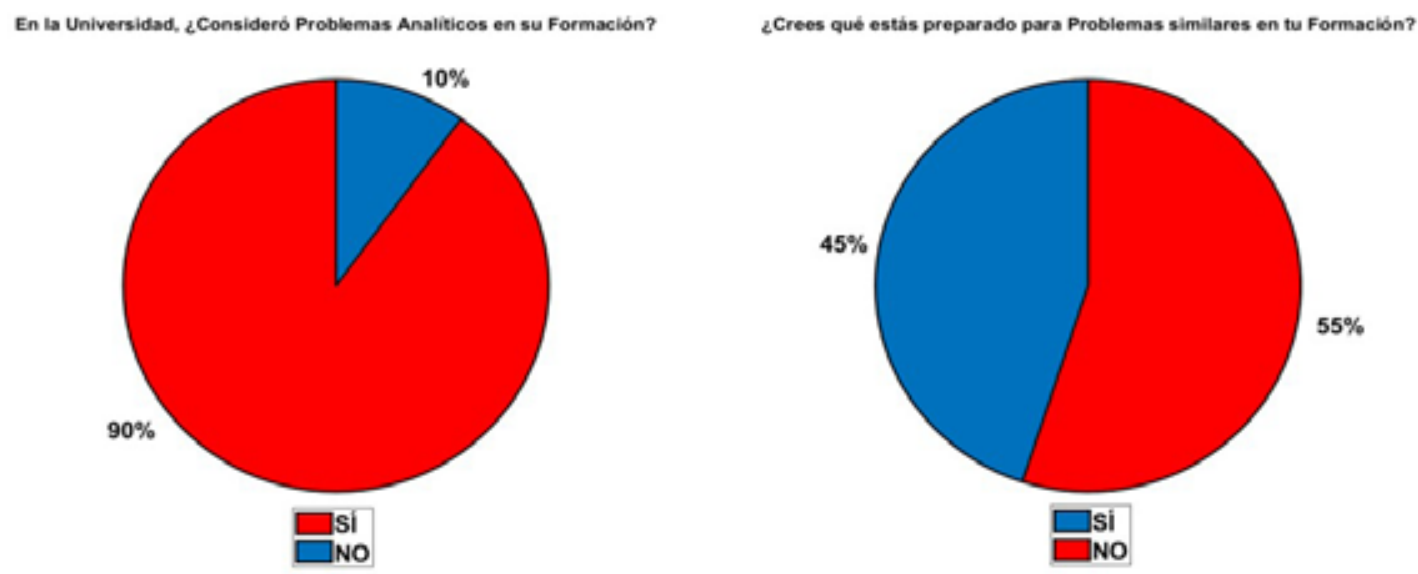

Figura 5. Percepción de problemas analíticos en la formación universitaria

La perspectiva de formación universitaria varía mucho sobre el enfoque de los cursos de Matemática a las carreras profesionales. Pero sin lugar a duda, unabuena formación académica permite una mejor perspectiva de la comprensión y la aplicabilidad de la matemática. Sin embargo como se pudo observar en la resolución, del problema propuesto, falta quizás más análisis en los problemas. Con lo cual, ante la evaluación por parte de los alumnos, en ese momento (abril), ellos indicaron que solamente un $45 \%$ estaría preparado para problemas similares, pero también surgió la consideración de problemas analíticos en su respectiva formación $90 \%$.
Finalmente, se mostraron los resultados de la última pregunta evaluada a su percepción a problemas similares del que se evaluó ese día, donde se obtuvo un $99 \%$ de aceptación de problemas analíticos en la formación porque ese tipo de exigencia ayudará a su preparación profesional (Figura 5). De forma adicional, la percepción de la pregunta que no se relaciona directamente con el enunciado generó confusión y rechazo debido a que usualmente con los datos que se brindan en el enunciado basta para resolver. Este hecho fue el punto clave del estudio para analizar si se analiza detalladamente un problema o es algo pragmático, actualmente, en la resolución de problemas. (Arias, 2005).

¿Qué es más importante en los Problemas Matemáticos para la Formación Académica?

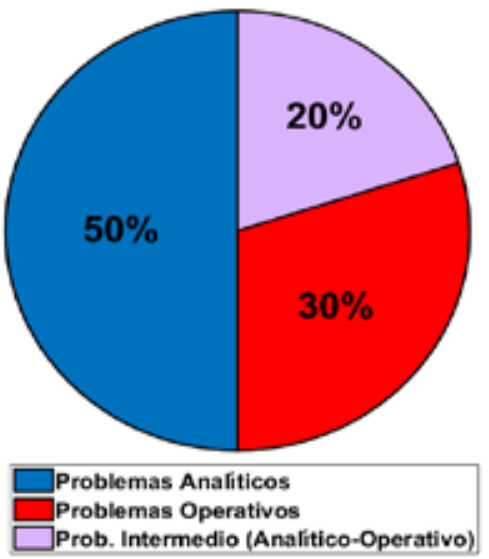

Figura 6. Nivel de importancia en un problema aplicado a la vida real 
Después de terminar el análisis, se solicitó una última respuesta con respecto a su apreciación del énfasis de los problemas universitarios que se dividió entre tres campos. De aquí, se obtuvo solamente un 30\% que se enfoca en problemas operativos, mientras que el $50 \%$ quiere que se enfoque en problemas analíticos. Pero hay un 20\% que desea una formación que se complemente ambos enfoques (Figura 6).

En este sentido, se logró satisfactoriamente los objetivos que se habían propuesto en el inicio de este estudio; el primero de evaluar el nivel análisis de un problema aplicado, al cual los estudiantes respondieron satisfactoriamente, y el segundo era observar el nivel de comprensión y análisis ante el problema propuesto que rompía el estilo de problemas que usualmente se utiliza en este tema de modelamiento matemático; y como un objetivo adicional sería la aceptación de este tipo de problemas donde se enfoque más el análisis del problema y definir correctamente las variables y el espacio de las soluciones. Sin lugar a duda, ha sido un estudio y aprendizaje compartido para los estudiantes evaluados y para nosotros.

\section{Comentarios de los estudiantes}

Realmente el estudio aplicado a los estudiantes fue una acción significativa para formalizar el proceso matemático con énfasis en el análisis, sin dejar de lado la parte operativa de los problemas para poder conocer y evaluar el impacto que tienen los problemas, y más aún, cuando la pregunta no guarda una relación supuestamente directa con el enunciado del mismo. Expondremos algunos comentarios de los estudiantes después de la realización y explicación del problema propuesto. (Aparicio \& Bazán, 1997). (Aparicio, 2006): (i) Cuando escuchaba el enunciado pensaba en la resolución hasta que escuché la pregunta, y no sabía qué hacer. Pensé que era una broma. (ii) Pensé que el problema era un chiste, porque no encontraba respuesta a la pregunta. (iii) Resolví rápidamente el ejercicio, y obtuve la respuesta negativa, y no supe que más hacer. (iv) Ha sido el problema más gracioso, pero también el más difícil de comprender para dar respuesta porque no esperaba la respuesta negativa y no sabía cómo relacionarlo con la pregunta.

(v) Aunque no pudiera resolver, el problema me hizo pensar mucho, porque no sabía cómo conectar los datos con la pregunta. Me gustó el planteamiento de la respuesta a partir de la edad negativa del hijo porque me hizo ver un análisis mucho más amplio. (vi) En el colegio nunca había realizado un problema parecido donde tenga que pensar de muchas formas para tener la respuesta, y creo que al resolver este problema la matemática me ayudará mucho más en la vida profesional. (vii) Este tipo de dificultades ayudan mucho más a considerar variables para resolverlos y no solamente que sean operativas donde se consiga la respuesta fácilmente. Me gustó mucho la explicación y la consideración del tiempo.

Sin lugar a dudas fue una actividad académica que suscitó preguntas y cuestionamientos a los estudiantes de cómo se deben relacionar los datos que brinda el problema, y cómo los datos se tienen que relacionar con la pregunta para brindar e interpretar la respuesta del problema que era el fin de esta 
aplicación en los estudiantes de primer año de la universidad. (Arias 1997; Malaspina 2016; Rodríguez 2016). Hay que considerar que este estudio se centró en cómo los estudiantes universitarios comprenden y analizan el enunciado del problema, y desde ahí, cómo lo resuelven y dan la respuesta a la pregunta del problema. También se enfocó en la perspectiva de lineamiento de los problemas matemáticos que necesiten una interpretación de comprensión lectora, y de ahí pasarlo al lenguaje matemático para ser desarrollado, y luego dar la respuesta con una adecuada interpretación al problema que refleja una interrogante de la vida real. (Aparicio, 2006) .

\section{Conclusiones}

Las matemáticas ayudan al estudiante a comprender la realidad mediante una situación problemática de tal forma que pueda construir una ecuación que respalde su razonamiento analítico, y así obtener respuestas a problemas de la vida real, lo cual exige la formación universitaria. La capacidad de análisis aprendido en el colegio (nivel secundario) según los estudiantes (450) que realizaron la prueba muestra que están más aptos a realizar problemas operativos que analíticos. A lo largo de nuestra experiencia docente, observamos que los estudiantes presentan más deficiencias en plantear el problema que en la parte operativa. El problema propuesto fue tomado con un factor gracioso, pero a la vez con una duda académica que no se esperaba la población estudiantil, debido que el enunciado de las hipótesis del problema no guardaba relación directa con la pregunta. Los estudiantes mostraron una crisis en cuánto a la pregunta del problema, y lo resolvieron mediante las ecuaciones, pero al obtener la solución de la ecuación no sabían cómo relacionarlo con la pregunta, y este hecho fue el eje principal del estudio. El problema propuesto permitió medir el nivel de comprensión y análisis de los estudiantes para realizar un modelado matemático aplicado a problemas reales. Solamente el hecho hacerles pensar en cómo relacionar las hipótesis con la pregunta ha sido el mayor logro del estudio. Los estudiantes comprendieron la importancia de un adecuado análisis del enunciado y formalización del espacio de las soluciones en donde el problema está incluido, además de la consideración de las unidades de cada variable. La formación universitaria debe ser más exigente en las formalidades para la construcción de los modelos matemáticos en los cursos de matemática a nivel superior debido que la comprensión de un problema real es más complicada de expresarla en ecuaciones. Si bien es cierto que la utilización de la tecnología en el aula en el proceso de la enseńanza-aprendizaje facilita al estudiante con respecto a los cálculos y entendimientos operativos del proceso; los docentes tenemos que hacer énfasis en nuestras sesiones de aula para reforzar la capacidad y actitud crítica e innovadora que tienen ellos 


\section{Referencias}

Aparicio , A., \& Bazán, J. (1997). Actitudes hacia las Matemáticas en ingresantes a la Universidad Nacional Agraria, La Molina. Más Luz, 351-380.

Aparicio. (2006). Las actitudes hacia la Matemática-Estadística dentro de un modelo de aprendizaje. Revista Educación, 15(28), 7-20.

Arias, N. (2005). Matemáticas en el Perú: un caso de responsabilidad social. Journal of Economics, Finance and Administrative Science, 10(18-19), 205-220.

Asensios, R. (2016). Rendimiento escolar en el Perú: Análisis secuencial de los resultados de la Evaluación Censal de Estudiantes. (B. C. R, Ed.) Serie de Documentos de trabajo, DT $N^{\circ} 2016-005$.

Baldor, A. (1941). Álgebra. Mexico: Grupo Patria.

Chuquilin Cubas, J. (2011). La educación secundaria en Perú y sus profesores: cambios y continuidades. Revista Educación, 95-117.

Cuena , R., Carrillo , S., De los Ríos, C., Reátegui, L., \& Ortiz, G. (2017). La calidad y equidad de la educación secundaria en el Perú. Instituto de Estudios Peruanos, IEP, 61-77.

Ecured. (2008). La habitación de Fermat. Recuperado el 12 de Noviembre de 2018, de Enciclopedia cubana:
https://www.ecured.cu/La habitaci\%C3\%B3n_de_Fermat

Flores, I. R. (2014). Situación actual de la Matemática en el Perú. Ensino e Cultura, 9(15), 82-95.

Gutiérrez, P. \&. (2011). Importancia de la matemática en el desarrollo del pensamiento científico $\mathrm{y}$ tecnológico. Investigación Educativa, 14(2), 67-78.

Jopen, G., Gómez, W., \& Olivera , H. (2014). Sistema Educativo Peruano: balance y agenda pendiente. Departamento de Economía PUCP, Lima, Lima.

Malaspina Jurado, U. (2016). Creación de problemas: sus potencialidades en la enseńanza y aprendizaje de las Matemáticas. Cuadernos de Investigación y formación en Educación Matemática, 11(15), 321-331.

(2014). Ministerio de Educación. Lima: Ley Universitaria Nº30220.

Pino. (2017). La Matemática conmo herramienta para entender la Economía dentro de la perpectiva de investigación de la Universidad Nacional Mayor de San Marcos. Pensamiento crítico, 22(1), 49-68.

Rodríguez. (2012). La enseñanza de las matemáticas en la crisis de la modernidad: por una renovación de la PAIDEIA. ORINOCO Pensamiento y Praxis, 2(1), 49-60. 
Rodriguez. (2016). La función social de la enseńanza de la matemática desde la matemática cotidianidad y pedagogía integral. ORINOCO Pensamiento y Praxis, 15(2), 34-45.

Tan Soo, T. (2018). Matemáticas Aplicadas a los Negocios, las ciencias sociales y de la vida (sexta ed.). España: Cengage learning.

Taylor, S., \& Bodgan, R. (1992). Introducción a los métodos cualitativos de investigación. Baidos.

Vilchez. (2007). Sistemas expertos para la enseñanza y el aprendizaje de la Matemática en la educaicón superior. Campo Abierto, 26(2), 83108.

Zapata \& Blanco, 2. (2009). Los estudiantes para profesores $\mathrm{y}$ sus concepciones sobre las matemáticasy su enseñanzaaprendizaje. 12(4), 109-122.

Zapata, M., \& Blanco , L. (2007). Las concepciones sobre las matemáticas y su enseñanza- aprendizaje de los profesores de matemáticas en formación. Cuadernos de Investigación y Formación en Educación Matemática, 2(3), 45-67. 
